\title{
Enjeux et perspectives des immunothérapies : un dossier entièrement réalisé par de jeunes oncologues
}

\author{
The Issues and Ways forward with Regards to Immunotherapies: a File Created Entirely \\ by Young Oncologists
}

\author{
C. Gervais \\ (C) Lavoisier SAS 2016
}

Pour ce numéro spécial, l'équipe de l'Association d'enseignement et de recherche des internes en oncologie (AERIO) a souhaité revenir sur les problématiques actuelles, translationnelles et fondamentales autour de la thématique « Immunologie et cancer ». Alors que le bouleversement des immunothérapies interroge les prises en charge de tous les acteurs en oncologie, le défi était de taille ! Ont donc répondu à ce projet huit internes guidés par des seniors bienveillants, avec le concours attentif de la revue Oncologie.

Trois questions principales ont été soulevées au cours de la conception de ce numéro : comment mieux prendre en charge les toxicités, quels moyens pour prédire et évaluer la réponse antitumorale et quelles seront les nouvelles indications à l'avenir?

La tolérance des immunothérapies est un sujet vaste et encore mal compris. A.Vozy et al. [1] se sont attachées à décrire la colite auto-immune, un des effets indésirables particulièrement fréquents, parfois limitants des inhibiteurs de checkpoints. De façon intéressante, un lien a été décrit récemment entre colite induite par anti-CTLA-4 et composition du microbiote [2]. En effet, une flore intestinale riche en bacteroides serait associée à un risque réduit de colite. Sur le versant endocrinien, T. Lambert et al. [3] se sont quant à eux intéressés aux dysfonctions thyroïdiennes, notamment des anti-CTLA-4 rapportées pour $7 \%$ des patients dans la littérature [4], ainsi qu'à leurs prises en charge. Les hypophysites concernent 1,8 à $3,3 \%$ des patients pour des doses de 1 à $3 \mathrm{mg} / \mathrm{kg}$ d'ipilimumab et deviennent particulièrement fréquentes lorsque la dose est supérieure $3 \mathrm{mg} / \mathrm{kg}$ (4,9 à $17 \%$ [5].

Les facteurs prédictifs de réponse antitumorale aux immunothérapies représentent une part importante des travaux actuels en recherche fondamentale, dont E. Colle et al. [6] ont réalisé une revue. Si le taux de réponse aux anti-PD-1 ou

C. Gervais $(\bowtie)$

Service d'oncologie-radiothérapie, hôpital de la Pitié-Salpêtrière, 47-83 Boulevard de l'Hôpital, F-75013 Paris, France e-mail : claire.gervais@aphp.fr
anti-PD-L1 est le plus souvent meilleur en cas de tumeur exprimant PD-L1, ce facteur ne peut être considéré comme prédictif pour le moment. Plusieurs problèmes se posent : difficultés techniques, variation selon le type histologique, hétérogénéité selon les compartiments. En effet, PD-L1 est davantage exprimé dans l'infiltrat tumoral que dans les cellules tumorales elles-mêmes [7]. L'évaluation même de cette réponse antitumorale pose question. E. Loir et al. [8] apportent des éléments de réponse dans leur article. Les critères immune-related response criteria (irRC) seraient mieux adaptés aux immunothérapies que les critères WHO et RECIST, car ils prennent en compte les progressions initiales et les réponses dissociées [9]. La pseudoprogression souvent évoquée ne représente que $4 \%$ des cas (principalement dans le mélanome, autour de 1-2\% dans la vessie et le rein) [10].

Concernant les nouvelles modalités d'immunothérapie, P. Gougis et al. [11] décrivent les virus oncolytiques avec notamment le talimogene laherparepvec ou T-VEC (un virus herpès simplex de type 1), validé dans le mélanome localement avancé ou métastatique et sans métastase viscérale, sur les résultats d'une étude de phase 3 [12]. La vaccination pose néanmoins plusieurs problèmes, notamment pharmacocinétiques (séquestration des virus dans le foie et la rate). Afin de mieux envisager les futures localisations, M. Tiako Meyo et al. [13] ont réalisé une revue pour les cancers urologiques avec quelques données de vaccination dans le cancer de la prostate, mais des résultats décevants des anti-CTLA-4 [14]. Les tumeurs testiculaires sont également un sujet d'étude prometteur de par leur forte expression de PD-L1, $73 \%$ des séminomes et $64 \%$ des TGNS [15]. Pour le cancer du sein triple-négatif, B. Verret et al. [16] ont rappelé que la présence de tumor infiltrating lymphocytes (TILs) était associée avec un meilleur pronostic et une diminution du risque de rechute à distance ou de décès [17]. Plusieurs essais précoces montrent des résultats encourageants en cas de maladie métastatique avec les anti-PD-1 et anti-PD-L1. Enfin, les tumeurs des voies aériennes et digestives supérieures (VADS) ont intéressé C. Gervais et al. [18], de par leur 
spécificité immunologique en cas d'infection par l'HPV. Avec l'arrivée du nivolumab en deuxième ligne métastatique réfractaire au platine dont les résultats ont été présentés à l'ASCO $2016^{\circledR}$ [19], difficile désormais d'oublier les cancers des VADS !

Nous espérons que ce numéro spécial de l'AERIO sera accueilli avec le même enthousiasme que les précédents et vous souhaitons une bonne lecture!

\section{Références}

1. Vozy A, Coutzac C (2016) Colite induite par les inhibiteurs de checkpoint immunitaire : anticorps anti-CTLA-4 et anticorps anti-PD-1/PDL-1. Oncologie 18:501-8

2. Dubin K, Callahan MK, Ren B, et al (2016) Intestinal microbiome analyses identify melanoma patients at risk for checkpointblockade-induced colitis. Nat Commun 7:10391

3. Lambert T, Doucet L (2016) Immunothérapie et dysfonctions thyroïdiennes : mise au point sur les connaissances actuelles. Oncologie 18:509-15

4. Hodi FS, O’Day SJ, McDermott DF, et al (2010) Improved survival with ipilimumab in patients with metastatic melanoma. N Engl J Med 363:711-23

5. Robert C, Thomas L, Bondarenko I, et al (2011) Ipilimumab plus dacarbazine for previously untreated metastatic melanoma. N Engl J Med 364:2517-26

6. Colle E, Andre T, Lebbe C, Borg C (2016) Facteurs prédictifs et biomarqueurs précoces de réponse aux inhibiteurs de checkpoint immunologiques (anti-PD-1, anti-PD-L1). Oncologie 18:533-42

7. Herbst RS, Soria JC, Kowanetz M, et al (2014) Predictive correlates of response to the anti-PD-L1 antibody MPDL3280A in cancer patients. Nature 515:563-7
8. Loir E, Jouinot A (2016) Immunothérapie et évaluation de la réponse tumorale : la difficulté de la pseudoprogression. Oncologie 18:523-6

9. Wolchok JD, Hoos A, O'Day S, et al (2009) Guidelines for the evaluation of immune therapy activity in solid tumors: immunerelated response criteria. Clin Cancer Res 15:7412-20

10. Chiou VL, Burotto M (2015) Pseudoprogression and immunerelated response in solid tumors. J Clin Oncol 33:3541-3

11. Gougis P, Teixeira L (2016) Les virus oncolytiques, première validation pour une classe thérapeutique pas si nouvelle. Oncologie 18:517-22

12. Andtbacka RHI, Kaufman HL, Collichio F, et al (2015) Talimogene laherparepvec improves durable response rate in patients with advanced melanoma. J Clin Oncol 33:2780-8

13. Tiako Meyo M, Huillard O, Alexandre J (2016) Immunothérapie en onco-urologie. Oncologie 18:527-32

14. Kwon ED, Drake CG, Scher HI, et al (2014) Ipilimumab versus placebo after radiotherapy in patients with metastatic castrationresistant prostate cancer that had progressed after docetaxel chemotherapy (CA184-043): a multicentre, randomised, doubleblind, phase 3 trial. Lancet Oncol 15:700-12

15. Fankhauser CD, Curioni-Fontecedro A, Allmann V, et al (2015) Frequent PD-L1 expression in testicular germ cell tumors. Br J Cancer 113:411-3

16. Verret B, Loirat D (2016) Immunothérapie et cancer du sein. Oncologie 18:551-8

17. Adams S, Gray RJ, Demaria S, et al (2014) Prognostic value of tumor-infiltrating lymphocytes in triple-negative breast cancers from two phase III randomized adjuvant breast cancer trials: ECOG 2197 and ECOG 1199. J Clin Oncol 32:2959-66

18. Gervais C, Scotté F (2016) Les cancers des voies aériennes et digestives supérieures à l'ère de l'immunothérapie : rationnels et spécificités de prise en charge. Oncologie 18:543-50

19. Ferris RL, Blumenschein GR, Fayette J, et al (2016) Further evaluations of nivolumab (nivo) versus investigator's choice (IC) chemotherapy for recurrent or metastatic $(\mathrm{R} / \mathrm{M})$ squamous cell carcinoma of the head and neck (SCCHN): CheckMate 141 - ASCO. J Clin Oncol 34 (suppl; abstr 6009) 